\title{
5GMEDE - 5G Mobile Edge Computing with Enriched Radio Network Information Services
}

\author{
A. Akman \\ Argela \\ Ankara \\ Turkey \\ Arda.Akman@argela.com.tr \\ J. Rodriguez \\ Instituto de Telecomunicações \\ Univerdidade de Aveiro \\ Aveiro, Portugal \\ jonathan@av.it.pt \\ A. Abdalla \\ Instituto de Telecomunicações \\ Univerdidade de Aveiro \\ Aveiro, Portugal \\ a.m.abdalla@av.it.pt
}

\author{
V. Monteiro \\ Departamento de Electrónica \\ Telecomuicações e Informática \\ Univerdidade de Aveiro \\ Aveiro, Portugal vmonteiro@ua.pt \\ P. Vieira \\ Instituto de Telecomunicações \\ ISEL/ADEETC, \\ Lisboa,Portugal \\ pedro.vieira@isel.pt
}

\author{
P. Marques \\ Escola Superior de Tecnologia \\ Instituto Politécnico Castelo Branco \\ Castelo Branco, Portugal \\ paulomarques@ipcb.pt \\ H. Marques \\ Escola Superior de Tecnologia, \\ Instituto Politécnico Castelo Branco, \\ Castelo Branco, Portugal \\ hugo@ipcb.pt
}

\begin{abstract}
G has a number of challenges to solve. Higher data usage and processing power necessities have emerged from the increasing number of mobile users as well as mobile applications becoming more and more demanding. There is a significant need to reduce the latency of the mobile network while cutting down on energy consumption. Backhaul traffic needs to be optimized to avoid setting up costly backhaul connections. Many IoT scenarios have conflicting requirements; the need for cheap and low complexity devices vs the need for processing power. Operators need to come up with value added services to avoid being dumb-pipe operators. The answer to these challenges and more require cloud-computing capabilities within the Radio Access Network (RAN) as well as a platform for mobile operators and third-party application providers to utilize these computing capabilities. This is where Mobile Edge Computing (MEC), one of the key emerging technologies for 5G, comes into play. The 5GMEDE project proposes to not only develop and demonstrate a complete MEC solution, including the MEC framework, Base Station services and applications to run on top, but also offers innovative features like constraint based mobile edge selection, using data analytics to enrich and refine real-time radio network information and utilizing Software Defined Wireless Networks (SDWN) concepts to improve mobility and resource allocation services to enable operators.
\end{abstract}

\section{Keywords-MEC, 5G, Energy Efficiency, RAN}

\section{INTRODUCTION}

Mobile Edge Computing (MEC) is first mentioned at the Mobile World Congress 2013 as a new network architecture concept that located increased computing resources at the very edge of the cellular network to locally deliver the content and the apps hosted on the platform. Designed to be implemented at the cellular base stations, MEC technology runs applications and performs processing tasks closer to the cellular user in order to reduce network congestion and enhance applications' performance. As it incorporates elements of telecommunications networking and information technology, MEC enables cellular operators to open their radio access network (RAN) to authorized 3rd parties including content providers and application developers. The concept proposed several benefits: with MEC, content and the apps could become more "aware" of customer locations and local network conditions, and they could be very low latency. Furthermore, MEC promises to optimize the backhaul links due to the ability to cache popular content to the customer. [1]

MEC technology is developed in response to the high network load and growing demand of network bandwidth in a landscape where cloud computing and $5 \mathrm{G}$ technologies are steadily gaining momentum. Within this context, MEC technology is already being considered as a key enabler towards $5 \mathrm{G}$ by the European Telecommunications Standards Institute (ETSI) which is also working on the concept's technical standards.

\section{5GMEDE FRAMEWORK}

IT and cloud-computing capabilities within the Radio Access Network (RAN) in close proximity to mobile subscribers are provided by Mobile Edge Computing (MEC). Since software-only entities which are running on a virtualized infrastructure that is found at or near the network edge, MEC enables implementation of mobile edge applications. MEC platform enables computation and storage capacity at the edge network therefore reduces network latency. The RAN edge offers applications and services that have context-related service capabilities which use direct access to real-time radio network information (such as subscriber location, cell load, etc.) and a service environment with ultra-low latency and high-bandwidth.

The environment as shown in Figure 1 provides proximity, ultra-low latency, high bandwidth, real-time access to radio 
network information, location awareness for innovation and value creation. These additional benefits can be leveraged by applications to create a new value chain, and new revenue streams.

While operators can generate new revenue streams through agreements with application or content providers, new value chains can be created for other players in the industry. Authorized third-party partners of operators including application and content providers can benefit from the Radio Access Network (RAN) edge by promptly deploying innovative services and applications towards mobile subscribers, enterprises and other vertical segments. The following parties can utilize proximity, context, agility, and speed benefits of MEC:

- Mobile operators

- $\quad$ Service providers

- Content providers

- Application providers

- $\quad$ Over the Top (OTT) players

- Independent Software Vendors (ISVs)

The project is intended to provide development of MEC framework that will cover system level and host level capabilities as well as enriched real-time radio network information from the base station. This framework will allow third-party applications to be deployed on MEC platform. The system will be demonstrated by use cases to prove operation in realistic scenarios.

To establish this framework the following functional elements will be developed during the 5GMEDE project:

- Mobile Edge System Level: includes ME hosts and ME management which are needed for running $\mathrm{ME}$ applications within an operator network and handles overarching view of multiple mobile edge hosts/probes/platforms (orchestrator)

- Mobile Edge Host Level: includes ME platform and a virtualization infrastructure which are designed to provide compute, storage, network resources and also contains the functions to run $\mathrm{ME}$ applications, and enable them to provide and consume ME services on Mobile Edge Platform and manages localized view of one mobile edge host/probe/platform such as platform manager and virtualization infrastructure manager

- Base Station Services: includes gathering real-time radio network information within the base station, measuring the energy consumption on multiple Base Station components using a data logger, enrichment of radio network information through data analytics, providing the raw and enriched radio network information to ME services

- Mobile Edge Applications: operates on virtualization infrastructure belonging to ME host and probes that are based on configuration or on request confirmed by ME management

A significant architectural goal of the project is to offer the application developers and content providers cloudcomputing capabilities and an IT service environment at the edge of the mobile network for creation of new services and new value chains.
Another goal of the project is to improve MEC concepts and services through innovative research and development activities, such as:

- Constraint based mobile edge selection by considering virtual resource requirements, latency requirements, on location requirements, etc.

- Using data analytics and forecast technologies to enrich real-time radio network information on the base station side, and providing refined radio information data to $\mathrm{ME}$ services and predicting network behaviour

- Utilizing SDWN concepts to improve MEC resource allocation services to meet mobility, flexibility and application requirements such as low latency, higher throughput

\section{A. Objectives and Motivation}

With the increasing number of mobile user equipment and applications becoming more demanding and requiring more resources, higher data usage and processing power necessities have emerged. Within 5G technologies, latency, energy consumption, and cost are needed to be minimized. In addition to this, it is required to decrease the backhaul traffic to avoid setting up costly high throughput backhaul connections. As a result of all of these requirements, the need for computational offloading, content optimization, content caching, etc. at the edge of the mobile network arise.

Through deployment of IT and cloud-computing capabilities within the Radio Access Network (RAN) and applications on the base stations, MEC targets providing solutions to the above problems. This is also inline with $5 \mathrm{G}$ vision of virtualization and software driven infrastructure.

With the convergence of IT and telecommunications networking and the evolution of mobile base stations, Mobile Edge Computing has become a natural development environment. For this reason, ETSI has established a MEC Industry Specification Group to define the standards in this area. Equally important European 5G PPP (5G Infrastructure Public Private Partnership) research body has recognized MEC, which is based on a virtualized platform, as one of the key emerging technologies for $5 \mathrm{G}$ networks, together with Network Functions Virtualization (NFV) and SoftwareDefined Networking (SDN). 5G networks not only define more advanced air interface technologies but also leverage more programmable approaches to software networking and use IT virtualization technology extensively within the telecommunications infrastructure, functions, and applications. MEC is among one of the key technologies and architectural concepts for that matter, as it helps advance the transformation of the mobile broadband network into a programmable world and contributes to satisfying the demanding requirements of $5 \mathrm{G}$ in terms of expected throughout, latency, scalability and automation.

The project proposes to solve above mentioned problems by developing the MEC framework and contribute to one of the key emerging technologies for $5 \mathrm{G}$. The importance of the project can be seen in the following table which provides a list of solutions that are currently not available: 


\begin{tabular}{|c|c|}
\hline $\begin{array}{l}\text { Upcoming } \\
\text { Requirements }\end{array}$ & Proposed Solution \\
\hline Minimize latency & $\begin{array}{l}\text { - } \text { Content caching } \\
\text { - Computational offload } \\
\text { - } \quad \text { Edge processing }\end{array}$ \\
\hline $\begin{array}{l}\text { High rate of data } \\
\text { processing }\end{array}$ & $\begin{array}{l}\text { - } \\
\text { - } \text { Content caching } \\
\text { - } \quad \text { Direct access to data } \\
\text { - } \\
\text { Data analytics }\end{array}$ \\
\hline $\begin{array}{l}\text { Low complexity } \\
\text { devices/Internet of } \\
\text { Things }\end{array}$ & $\begin{array}{l}\text { - } \begin{array}{l}\text { Off-loading compute } \\
\text { capacity to the mobile } \\
\text { edge host }\end{array} \\
\text { - Additional storage } \\
\text { capabilities } \\
\text { - Remote ME radio network } \\
\text { benchmark probes and } \\
\text { energy probes }\end{array}$ \\
\hline $\begin{array}{l}\text { Operator value added } \\
\text { services }\end{array}$ & $\begin{array}{l}\text { - Utilizing application data } \\
\text { available at the mobile } \\
\text { edge host and probes } \\
\text { - Data analytics and } \\
\text { forecasting services } \\
\text { - Utilizing radio } \\
\text { information such as } \\
\text { getting UE location, } \\
\text { upload and download } \\
\text { throughput, RSSI, base } \\
\text { station utilization, etc. }\end{array}$ \\
\hline $\begin{array}{l}\text { Storing a large amount } \\
\text { of data locally }\end{array}$ & $\begin{array}{ll}\text { - } & \begin{array}{l}\text { Additional storage } \\
\text { capabilities }\end{array} \\
\text { - } & \text { Direct access to data }\end{array}$ \\
\hline $\begin{array}{l}\text { Significant savings in } \\
\text { backhaul capacity }\end{array}$ & $\begin{array}{l}\text { - Content caching (Mobile } \\
\text { edge application stores the } \\
\text { most popular content } \\
\text { consumed in the } \\
\text { geographical area locally) } \\
\text { - Computational offload } \\
\text { - Edge processing }\end{array}$ \\
\hline
\end{tabular}

\section{Business PERSPECTIVE}

The mass adoption of pervasive digital technologies and increased utilization of data-intensive applications on mobile broadband networks result in an explosion of data traffic due to the dramatic acceleration of mobile data, audio and video consumption. The GSMA 2016 report forecasts data traffic volumes to grow at a CAGR of $49 \%$ over the next five years - a more than seven-fold increase - approaching 40 EB per month by 2020. This means the global average of data usage per subscriber per month will reach to $7 \mathrm{~GB}$. The rising data usage per subscriber is expected to decrease Mobile Network Operators' (MNOs) profit margins while forcing them to take action towards evolving their asset portfolios and business models to meet the rising demand. The biggest business challenge for global operators in the years to come is seeking new revenue generation opportunities and diminishing the cost of data delivery while trying to scale their network capacity.

As envisioned by GSMA Intelligence, the mobile market is expected to witness an exponential growth of Internet of Things (IoT) for the coming years, reaching to over 50 billion connected devices by 2020 . By 2025 , the total global worth of IoT technology could be as much as USD 6.2 trillion. As IoT devices and applications continue to attract more and more mobile subscribers while expanding their market share, they will generate a vast amount of mobile data which will surely overwhelm the mobile networks unless MNOs carefully manage the network resources. Looking from this perspective, MNOs might have no chance but to invest on the infrastructure to satisfy this growing demand in the following years.

In this context, Mobile Edge Computing (MEC) emerges to help MNOs by reducing the cost of data delivery, improving user experience, creating new revenue generating services, and letting MNOs to fully expand to new markets like IoT. As identified by ETSI, the MEC standard specifies the integration of an NFV-based cloud computing environment within the mobile radio access network (RAN). Whilst challenging the traditional architectures where computational power is centralized at the core network, the concept of MEC aims to bring cloud computing capabilities closer to mobile subscribers. By providing these capabilities in close proximity to end-users, MEC promises to meet high-bandwidth delivery and ultra-low latency demands of future IoT applications.

\section{A. European Perspective}

Currently, the majority of data traffic is generated in North America and Europe, where an average mobile user consumes 4.4 GB and 1.8 GB per month respectively [2]. As foreseen in GSMA Intelligence Report, by 2020 the average North American and European subscriber is expected to consume around $22 \mathrm{~GB}$ and $12 \mathrm{~GB}$ of mobile data per month respectively, as can be seen in figure 2 . With the rising rates of IoT popularity, European subscribers will likely adopt the concept IoT and vastly incorporate smart objects and smart home devices to their lives. Besides, based on the high rates of $4 \mathrm{G}$ adoption in the European mobile market, the nextgeneration $5 \mathrm{G}$ networks are promising to be vastly deployed in the Europe by the time $5 \mathrm{G}$ standards are fully determined.

\section{B. National perspective for PORTUGAL}

The mobile telecommunications industry deals with countless expenses, both operational (OPEX) and capital (CAPEX). The sky-rocketing technological evolution at the consumer side based on a massive exchange of data, needs a highly available network framework with abundant resources. This is possible due to new and more efficient Radio Access Technologies (RAT)s, and improved efficiency of communication between the Radio Access Network (RAN) and the network core. Currently, most network providers operate simultaneously with different RATs, at different 
frequency bands, leading to the Heterogeneous Wireless Network (HWN) concept. More, the need for higher capacity, to fulfil the users eager for data connectivity, has led the operators to plan the network with even higher site density, increasing the costs.

Delivering mobile services to several billion users around the world comes with a cost of energy consumption and $\mathrm{CO} 2$ emissions, especially in the RAN, where thousands of basestations are deployed, which represent up to $60 \%$ of the total network energy consumption.

Hence, the scenario of accurately measuring the RAN consumption is drawing a lot of attention to the operators, not only overall, but also in a more detailed way, by measuring the specific energy consumption at the main elements that build the base-station, from the radio, to the controller or combiner. Furthermore, network statistics are available, allowing to measure the main Key Performance Indicators (KPIs) in the different scenarios and technologies.

\section{Visible Results and Products}

Project will demonstrate how MEC can minimize latency, reduce backhaul traffic, reduce download times, improve media quality, enables operators and third party service providers to come up with new services which as a result not only increase user satisfaction but also generate new revenue streams and save CAPEX and OPEX for the telecommunications ecosystem.

Along with the demonstration of MEC capabilities and benefits, the final outcome of the project will be a complete MEC solution which includes MEC framework, base station services and MEC applications.

The base station services include measuring the energy consumption on multiple Base Station components, from the radio, to the controller or combiner. This will enable detailed energy consumption measurements and evaluating the impact of MEC in base station operation.

A test bed will be setup during the project to experiment and develop prototypes. Two different test setups will be established, one version being a remote, distributed test bed with each partner using their equipment locally and then a second version where all the equipment is brought together in one of the partners labs. In addition, this test bed will also be used for demonstration purposes, especially for the content caching application demonstration.

As ARGELA is the R\&D company of Turk Telekom, it does have access to Turk Telekom test network. The consortium is planning the use this opportunity to demonstrate the project outcome on Turk Telekom test network.

Along with the product development effort will be made on standards activities. Since the consortium is very active in ETSI MEC Industry Specification Group (ISG) standardization activities, consortium will try to contribute project results. This standardization effort is discussed in detail in the following section.

All partners may pursue IPR rights on their research activities throughout the project. ARGELA has been actively filing patents on programmable and virtualizable base stations

\section{TESTING AND DEMONSTRATIONS}

Including in the demonstration scenario of the 5GMEDE is related to the Enriched Radio Network Information Services, more specifically, innovative solutions to monitor and reduce the energy consumption at the mobile radio network base stations, including the measurement of network key performance indicators to estimate how the end-user Quality of Experience (QoE) is impacted by the new 5G functionalities (such as MEC) and different mobile radio network configurations [3]. The 5GMEDE project will be built on top of an existing network management platform, brought to the project by CELFINET partner, which is to be significantly improved to increase its market value in the context of the upcoming $5 \mathrm{G}$ system.

It is in this context that it is essential to optimize the energy consumption of the grid radio, as it is a crucial factor in reducing OPEX. On the other hand, the constant migration radio technology $(2 \mathrm{G}, 3 \mathrm{G}, 4 \mathrm{G}$ and more recently $5 \mathrm{G})$ have brought new features that are implemented on the network, often without analyzing the real impact on energy efficiency and return on investment (ROI) for the operator mobile. For example, one of the emerging features for $5 \mathrm{G}$, is Mobile Edge Computing (MEC), which consists of reducing backhaul traffic when using capabilities of cloud computing within the radio access network and applications at stations base, considerably reducing latency when placing content closer to the user. However, the real impact of this new feature on consumption energy is not yet studied.

The energy performance of the mobile network plays an especially important role at this moment of transition from $4 \mathrm{G}$ to $5 \mathrm{G}$. Proof of this is that in the definition of technical requirements of $5 \mathrm{G}$, mobile operators aim to reduce of the total energy consumption of the network by $50 \%$, even for a scenario of 1000 times mobile traffic [4], where at same time on mobile networks, the data traffic generated in the last two years increased by 7\%, reaching 3.7 exabytes per month [5].

It therefore becomes increasingly important for the mobile operator to be aware of the energy consumption of your radio network in real time, not only globally, but mainly at the level of specific consumption in the main elements that make up each base station. With this approach, the operator will have energy consumption data available that reflect the operation of the grid in different scenarios / configurations and technologies, allowing their optimization energy through Machine Learning algorithms in a logic of self-learning and self-configuration (SON- Self-Organizing Network).

This need represents an important business opportunity for companies 5GMEDE consortium companies, through the sale of innovative solutions to mobile operators to help them migrate to $5 \mathrm{G}$, reducing consumption and improving the end user's QoE (Quality of Experience) (increased transmission rate, reduced latency, etc.).

\section{CONCLUSIONS}

In the context of the 5GMEDE project, we will address the issue of developing and demonstrate a complete MEC solution, including the MEC framework, Base Station services and applications to run on top, and also offers innovative features like constraint based mobile edge selection, using data analytics to enrich and refine real-time radio network information and utilizing Software Defined Wireless 
Networks (SDWN) concepts to improve mobility and resource allocation services to enable operators and applications providers to come up with new and improved service capabilities. With advanced technologies and architectural concepts MEC provides computation and storage capabilities in close proximity to mobile subscribers and offer applications and services with context-related service capabilities to have direct access to real-time radio network information and a service environment with ultra-low latency and high bandwidth, one of main challenge that $5 \mathrm{G}$ aims to solve.

\section{ACKNOWLEDGMENT}

This work was carried out under the 5GMEDE project $\mathrm{N}$. 33583 - co-financed by the European Funds (FEDER) by PT2020 of ANI.
[1] Dyer, By Keith. "On the Edge: The Story of Mobile Edge Computing." The Mobile Network On the Edge the Story of Mobile Edge Computing Comments. Web

[2] "The Mobile Economy 2016." GSMA Intelligence, Web. https://www.gsmaintelligence.com/research/?file=97928efe09cdba28 64cdcflad1a2f58c\&download

[3] E. Vesterinen, "Linking QoE and QoS on data services", Novembro de 2014. [Online]. Available in https://www.itu.int/en/ITUT/Workshops-and-

Seminars/qos/112014/Documents/Presentations/S5P1-Esa-V2.pdf [From May 2017].

[4] NGMN Alliance, February 2015, NGMN 5G White paper

[5] Cisco, "Cisco Visual Networking Index: Global Mobile Data Traffic Forecast Update, 2015" White Paper", Feb 2016, [Online]. Available in http://www.cisco.com/c/en/us/solutions/collateral/serviceprovider/visual-networkingindex-vni/mobile-white-paper-c11520862.html [Consultado em Maio 2017].

\section{REFERENCES}

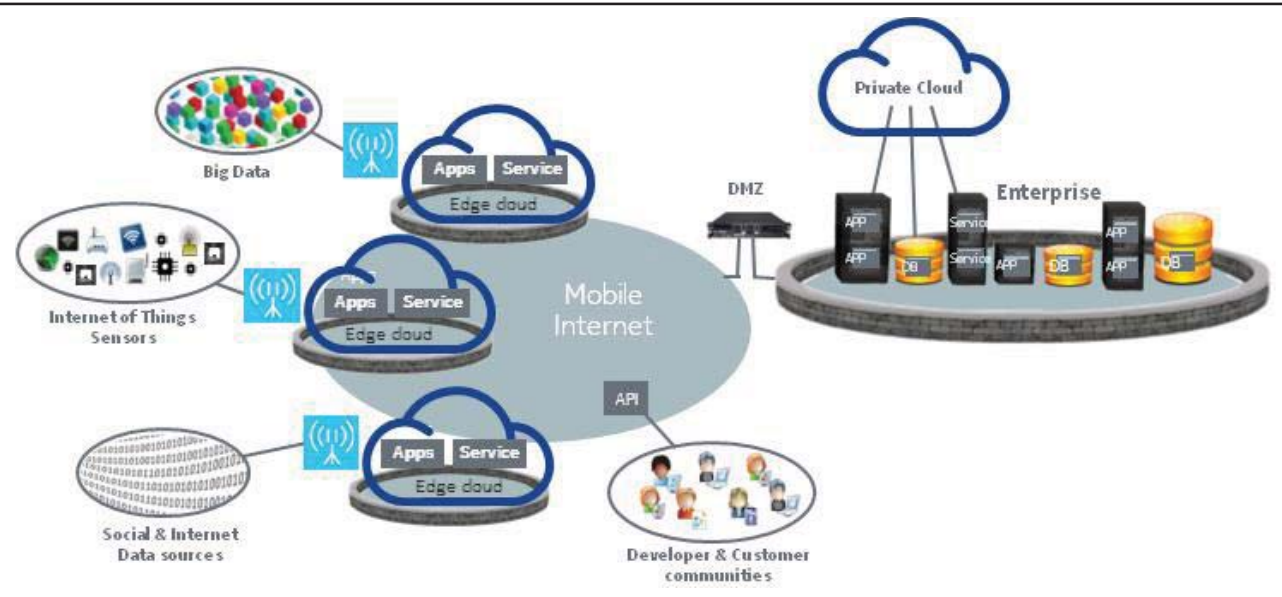

Fig. 1. MEC environment

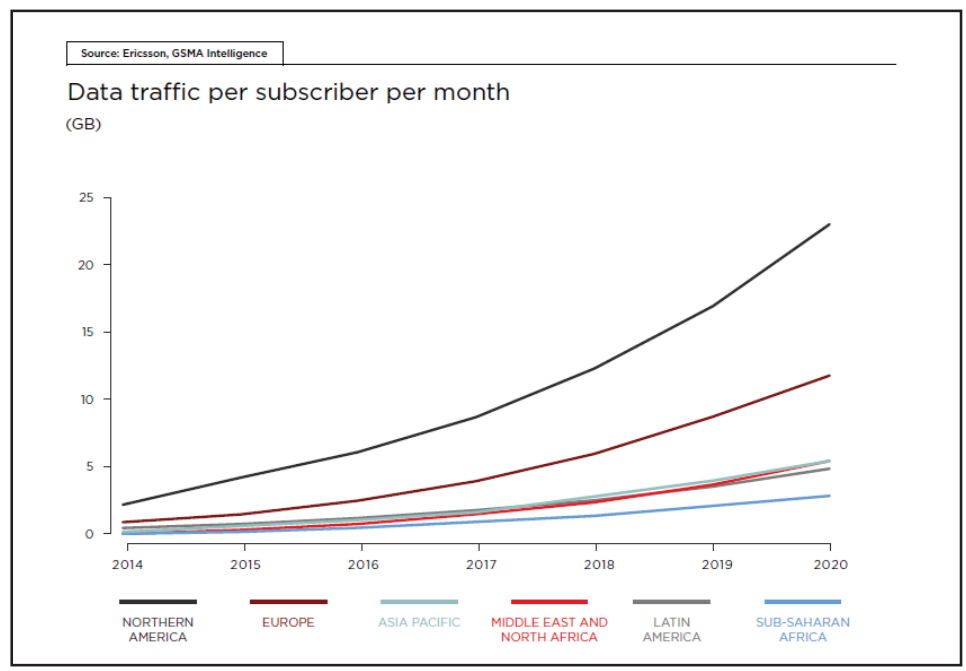

Fig. 2. Data Traffic per Subscriber per Month 